\title{
Identifying a Whole-brain Connectome-based Model in Drug-naïve Parkinson's Disease for Predicting Motor Impairment
}

\section{Haoting Wu}

Zhejiang University School of Medicine Second Affiliated Hospital

\section{Cheng Zhou}

Zhejiang University School of Medicine Second Affiliated Hospital

\section{Tao Guo}

Zhejiang University School of Medicine Second Affiliated Hospital

\section{Jingjing Wu}

Zhejiang University School of Medicine Second Affiliated Hospital

\section{Xueqin Bai}

Zhejiang University School of Medicine Second Affiliated Hospital

Xiaocao Liu

Zhejiang University School of Medicine Second Affiliated Hospital

Jingwen Chen

Zhejiang University School of Medicine Second Affiliated Hospital Jiaqi Wen

Zhejiang University School of Medicine Second Affiliated Hospital

\section{Xiaojun Guan}

Zhejiang University School of Medicine Second Affiliated Hospital

\section{Ting Gao}

Zhejiang University School of Medicine Second Affiliated Hospital

\section{Luyan Gu}

Zhejiang University School of Medicine Second Affiliated Hospital

\section{Peiyu Huang}

Zhejiang University School of Medicine Second Affiliated Hospital

\section{Xiaojun Xu}

Zhejiang University School of Medicine Second Affiliated Hospital

\section{Baorong Zhang}

Zhejiang University School of Medicine Second Affiliated Hospital

\section{Min-Ming zhang ( $\nabla$ zhangminming@zju.edu.cn )}

The 2 nd affiliated hospital of zhejiang university, school of medicine https://orcid.org/0000-00030145-7558 
Research

Keywords: brain connectome, resting-state fMRI, Parkinson's disease, motor impairment, predict

Posted Date: August 10th, 2021

DOl: https://doi.org/10.21203/rs.3.rs-779169/v1

License: (c) (i) This work is licensed under a Creative Commons Attribution 4.0 International License.

Read Full License 


\section{Abstract \\ Background}

The functional alternation of distinct brain networks contribute to motor impairment in Parkinson's disease (PD) remains unclear. Identifying a whole-brain connectome-based predictive model (CPM) in drug-naïve patients and verifying its predictability among drug-managed patients would be helpful to detect generalizable brain-behavior association and reflect intrinsic functional underpinning of motor impairment.

\section{Methods}

Resting-state functional data of 47 drug-naïve patients were enrolled to construct a predictive model by using the CPM approach, which was subsequently validated in 115 drug-managed patients. The severity of motor impairment was assessed by calculating Unified Parkinson's Disease Rating Scale part III (UPDRS III) scores. Predictive performance was evaluated with the correlation coefficient $\left(r_{\text {true }}\right)$ and the mean squared error (MSE) between observed and predicted scores.

\section{Results}

A CPM for predicting individual motor impairment in drug-naïve PD was identified with significant performance $\left(r_{\text {true }}=0.845, p<0.001, M S E=137.57\right)$. Two connection patterns were recognized according to the correlation coefficients between the connections' strength and motor impairment severity. The negative motor-impairment-related network contained more within-network connections in the motor, visual-related, and default mode networks, while the positive motor-impairment-related network was constructed mostly with between-network connections coupled motor-visual, motor-limbic, and motorbasal ganglia networks. The predictability of constructed model was further confirmed in drug-managed patients $(r=0.209, p=0.025, M S E=182.96)$, suggesting generalizability in PD patients with lasting dopaminergic medication influence.

\section{Conclusions}

This study identified a whole-brain connectome-based model that could predict the severity of motor impairment for PD. The connection patterns generated from the model reflected that functional segregation of motor, visual-related, and default mode networks play an important role in PD motor impairment, and higher connections coupling motor and non-motor regions might demonstrate a compensatory mechanism to overcome motor impairment.

\section{Background}


Parkinson's disease (PD) is the second most common neurodegenerative disease in aging populations[1], which in its most classical manifestation is characterized by progressive motor impairment [2]. It is demonstrated that motor impairment in PD results from abnormalities of the basal ganglia circuits due to the death of dopaminergic neurons in substantia nigra pars compacta [3], however, pathophysiological changes beyond basal ganglia have been widely acknowledged, that are also playing significant roles in modulating motor loops [3-5]. Moreover, complicated within-network segregation and between-network coupling may bring significant contributions to motor disorder; but, in such a large-scale brain network view, the potential network underpinning for PD is not well investigated, and little is known to the exquisite interactions among/within distinct networks.

In past decades, resting-state functional magnetic resonance imaging (rs-fMRI) provides an approach for studying central processing of motor impairment in vivo. As significantly documented, rs-fMRI studies have successfully identified association between motor impairment and functional connections in classic motor regions such as basal ganglia and motor cortex[6, 7], as well as non-motor regions including frontoparietal network, visual networks and limbic system [8-11]. However, these findings obviously vary from each other. One of the main concerning is that, in the recruitment, PD patients have already exposed to dopaminergic medication, which would lead to heterogenous reorganizations of brain function in order to preserve motor behavior $[12,13]$. Such important influence is widely ignored. Therefore, we hypothesized that the exploration in drug-naïve patients would be a priority of disclosing intrinsic exquisite interactions among/within district networks relating to motor impairment, which may be robust in predicting motor impairment when medication influence is subsequently taken into consideration.

Moreover, because the neurodegenerative process for human brain is not always consensus, but with different trajectories and confounded by various pathophysiological factors, more insight has been paid to the personal brain organization. Detecting the link between individual functional connectome and behavioral measurements can maximally reduce the bias brings from population variation, and the observed brain-behavior associations would increase robustness and generalizability[14]. Thus, a newly applied connectome-based predictive modeling (CPM) approach has been introduced to predict behavior at the individual level by using large-scale network functional connectivity in a machine-learning framework, which has been employed to explore complicated mechanisms in mental and cognitive disorders [15-17], as well as in predicting outcome after deep brain stimulation in PD patients[18]. Therefore, by taking advantage of CPM framework built on informative large-scale network connections, a novel predictive model would be constructed to identify intrinsic network patterns for drug-naïve patients, which may have robust performance in predicting motor impairment.

Hence, this study aimed to construct a whole-brain connectome model that can predict motor impairment for PD patients. In order to reveal the disease-intrinsic functional underpinning without any effect of dopaminergic medication, we constructed a predictive model on drug-naïve patients and further tested its performance in independent drug-managed PD patients with the purpose of reliability checking.

\section{Methods}




\section{Participants' enrollment and evaluation}

All patients signed informed consent forms in accordance with the approval of the Medical Ethics Committee of the Second Affiliated Hospital of Zhejiang University School of Medicine.

A total of 200 PD patients were initially recruited in this study. The diagnosis of PD was made by a senior neurologist (B.R.Z.) according to the United Kingdom PD Society Brain Bank criteria [19]. Twenty-four patients were excluded since they had: (1) cerebrovascular disorders, including previous stroke, history of head injury, and other neurologic diseases ( $N=14)$; (2) cognitive impairment based on the Mini-Mental State Examination (MMSE) estimated by the criteria suitable for Chinese population (MMSE score $\leq 17$ for illiterate patients, $\leq 20$ for grade-school literate, and $\leq 23$ for junior high school and higher education literate $(N=10)[20,21]$. Finally, 176 PD patients were enrolled in this study, including 49 drug-naïve patients and 127 drug-managed patients. For drug-managed PD patients, clinical assessments were performed in the morning after withdrawing all dopamine replacement therapy overnight (at least $12 \mathrm{hrs}$., on their "drug-off status"). Basic demographic information, including age, sex, education, and disease duration, and neurologic and psychiatric scales including Unified Parkinson's Disease Rating Scale (UPDRS) part III score, Hoehn and Yahr stage (H-Y stage), and MMSE score were obtained from all patients. The total levodopa equivalent daily dose (LEDD) [22] and duration of treatment were recorded for drug-managed patients.

\section{Image acquisition and preprocessing}

\subsection{Image acquisition}

All imaging data were acquired from a 3.0 Tesla magnetic resonance imaging (MRI) scanner (Discovery MR750; GE Healthcare). MRI scanning for each drug-managed patient was carried out in drug-off status. Head of each participant was stabilized with foam pads, and earplugs were provided to reduce the noise during scanning.

Resting state functional MRI (rs-fMRI) data were acquired using Gradient Recalled Echo - Echo Planar Imaging sequence: $\mathrm{TE}=30 \mathrm{~ms} ; \mathrm{TR}=2,000 \mathrm{~ms} ; \mathrm{FA}=77$ degrees; $\mathrm{FOV}=240 \times 240 \mathrm{~mm}^{2} ;$ matrix $=64 \times 64$; slice thickness $=4 \mathrm{~mm}$; slice gap $=0 \mathrm{~mm}$; number of slices $=38$ (axial); time points $=205$. Structural T1weighted images were acquired using a Fast Spoiled Gradient Recalled sequence: echo time $=3.036 \mathrm{~ms}$; repetition time $=7.336 \mathrm{~ms}$; inversion time $=450 \mathrm{~ms}$; flip angle $=11$ degrees; field of view $=260 \times 260$ $\mathrm{mm}^{2}$; matrix $=256 \times 256$; slice thickness $=1.2 \mathrm{~mm}$; number of slices $=196$ (sagittal). All the sequence FOVs covered the whole brain, including cerebrum, cerebellum and brain stem.

\subsection{Image preprocessing}

Rs-fMRI data processing was carried out using Statistical Parametric Mapping (SPM 12, https://www.fil.ion.ucl.ac.uk/spm/) and Data Processing Assistant for Resting State fMRI (DPABI_V3.1_180801, http://www.rfmri.org/) [23]. At the beginning, the first 10 volumes of functional time 
series were deleted letting MRI signal to reach the equilibrium. The remaining images underwent slice timing for interval scanning, realignment, and normalizing to the standard MNI space through T1 images. Next, spatial smoothing with a Gaussian kernel of $6 \times 6 \times 6 \mathrm{~mm}$ full-width-at-halfmaximum, detrending, covariates regression (Friston 24-motion parameters) and band-pass temporal filtering $(0.01-0.1 \mathrm{~Hz})$ were subsequently applied to the remaining volumes.

\subsection{Head-motion control}

Considering the effect of head-motion on the rs-fMRI analysis, an additional 14 patients including 2 drugnaïve and 12 drug-managed patients with excessive head-motion ( $2 \mathrm{~mm}$ in displacement and 2 degrees in rotation and mean frame-wise displacement (FD) $0.2 \mathrm{~mm}$ ) were excluded [24, 25]. After that, a total of 162 PD patients were enrolled in this study, including 47 drug-naïve and 115 drug-managed patients. To verify that neither observed nor predicted scores were correlated with head-motion, the correlation coefficients were calculated between the mean FD and observed and predicted score, respectively. To further control the possible head-motion effect, we also applied additional prediction analysis with the mean FD as an additional nuisance variable within the candidate connections selection process described in part 3.1 (candidate connections selection by using LOOCV procedure).

\subsection{Functional network construction}

Consistent with previous studies applying CPM, network nodes were defined using the 268-ROI functional brain atlas[26]. This atlas covers the whole brain, including cortical, subcortical, and brainstem structures. The whole-brain functional connection matrix was constructed for each patient using GRETNA [27]. The mean time series of each node was extracted by averaging the time series of all voxels in each defined node. And then the functional connection is calculated as the Pearson-correlationcoefficient ( $r$ ) between the mean time series of each pair of nodes. A Fisher's r-to-z transformation was then used to normalize the correlation coefficients, and the resulting $268 \times 268$ matrix for each participant was utilized for the following CPM analysis. Each element of the matrix represented the strength of connection between two nodes.

\section{Connectome-based model construction and evaluation in drug-naïve patients}

The flowchart of connectome-based model construction and evaluation was shown in figure 1.

\subsection{Selection of candidate connections by using LOOCV procedure}

Considering the relatively small number of drug-naïve patients $(\mathrm{N}=47)$, the leave-one-out cross-validation (LOOCV) procedure was used to select candidate connections [28, 29]. The LOOCV procedure was repeated iteratively. In each iteration, one patient was removed from the training set and data of the remaining N-1 patients was used for testing through the following steps. First, the correlation between the strength of each connection and the observed UPDRS III score was assessed. In this step, recommended spearman's analysis was applied since the distribution of observed scores in this study did not follow the normal pattern (Kolmogorov-Smirnov test, $\mathrm{p}<0.05$ )[30]. Besides, partial correlation analysis was applied to 
minimize the possible confounding effects of nuisance variables, including age, sex, and disease duration. Next, an optimal threshold was applied to select candidate connections that were significantly correlated with UPDRS III scores (detailed in part 3.4 Optimal threshold exploration). Finally, all selected connections were split into positive connections (connections whose strength indexed higher UPDRS III score and severe motor impairment) and negative connections (connections whose strength indexed lower UPDRS III score and mild motor impairment) according to their correlation coefficients with observed scores. The above-mentioned steps would be repeated for $\mathrm{N}(\mathrm{N}=47)$ times until all patients had been left out.

\subsection{Model construction with consensus connections and predictability evaluation}

After LOOCV procedure, forty-seven sets of candidate connections would be obtained. Because of the nature of cross-validation, a slightly different set of candidate connections may be selected in different iterations. To reduce potential variation, the connections that were finally enrolled for model construction should be selected in each iteration, termed consensus connections. These connections had the highest reliability among all candidate connections. Then, the strength of each positive and negative consensus connection was summed, respectively. And strength sums of positive and negative consensus connections were then fitting into general linear regression to build a relationship with the observed score. The predicted score of each patient could be calculated by applying the constructed linear model with the following formula:

predicted score $=a_{1} \times x_{1}+a_{2} \times x_{2}+b$

$\left(x_{1}\right.$ : sum of strength of consensus positive connections, $x_{2}$ : sum of strength of consensus negative connections)

The performance of the constructed model was evaluated by calculating the Spearman correlation coefficient $\left(r_{\text {true }}\right)$ and the mean squared error (MSE) between observed and predicted UPDRS III scores. The value of correlation coefficient and MSE are usually dependent, higher correlation implies lower MSE and vice versa. A lower MSE value means a small difference between predicted and observed score [30]. The significance of the constructed model was further tested by applying the 1000-permutation-test [15]. This test was done by randomly shuffling the UPDRS III score along with repeating the above processes 1000 times. The significance of the permutation test was analyzed by calculating the percentage of sampled permutations that are greater or equal to the $r_{\text {true }}\left(p_{\text {permu }}\right), p_{\text {permu }}<0.05$ was considered as statistically significant.

\subsection{Predictability comparison among models constructed using different methods}

Before external validation, it is necessary to ensure that the model (M) constructed within the above processes obtaining the best predictability in drug-naïve patients. Therefore, three models constructed with different methods were employed, including model M1 constructed with consensus positive connections, model M2 constructed with consensus negative connections, and model M3 generating 
predicted scores from each iteration of LOOCV. The construction processes of three models (M1, M2, and M3) were detailed in supplemental materials (Title: construction processes of $M 1, M 2$, and M3). To explore whether the predictability of these models was significantly different from model $M$, the Steiger's

$Z$ test was applied to compare the $r_{\text {true }}$ of model $M$ and the other three models [29]. The optimal threshold exploration and permutation test were also applied during the construction of M1, M2, and M3. Thus, the thresholds of these four models can be different.

\subsection{Optimal threshold exploration}

For candidate connections selection, rather than applying an arbitrary $p$-value threshold as previous study [29], predictability comparison was employed among different p-values cutoffs. These thresholds were explored by repeating the above process 50 times using $p$ values ranging from 0.05 to 0.001 , with an interval of 0.001 each time. The $p$-value that leads to the highest $r_{\text {true }}$ was selected and employed in the subsequent model construction.

\section{Model validation in drug-managed patients}

Finally, the model with the best predictability among the above four ones was further validated in drugmanaged patients. Correlation coefficient $(r)$ and MSE between observed and predicted score were also calculated. The significance of $r$ was calculated using standard parametric conversion and $p<0.05$ was considered as statistically significant.

\section{Functional networks anatomy of constructed connectome model}

\subsection{Grouping nodes into seven functional networks}

These 268 nodes were divided into seven canonical functional networks according to the anatomical order and previous studies [26, 31], which include frontoparietal (63 nodes), default mode (20 nodes), motor ( 50 nodes), visual-related ( 45 nodes), limbic ( 30 nodes), basial-ganglia ( 29 nodes) and cerebellum (31 nodes) networks. The visual-related network includes visual I, visual II, and visual association networks. Map of these seven networks was shown in the supplementary material (Figure S1). Connections within each network (within-network connection) were calculated by summarizing the number of connections that connecting nodes among it, and connections between two distinct networks (between-network connection) were calculated by summarizing the number of connections that coupled nodes from them.

\subsection{Contribution of each functional network}

We weighed each functional network's contribution by calculating the sum of consensus positive and consensus negative connections that belong to it, and a higher number of connections indicates a greater contribution. To further access the importance of each functional network to the motor impairment prediction, we computationally "lesioned" the model to exclude connections from it. That is, in an iterative analysis, we masked the connection matrix to exclude connections that appeared in one of the seven 
functional networks. For example, after excluding connections in the frontoparietal networks, which contained 63 nodes, a 205205 matrix rather than 268268 matrix was submitted for analysis. Models with lesioned matrices, which are defined as lesioned models, were first constructed and evaluated among drug-naive patients, then they were validated among drug-managed patients. The predictive ability of each "lesioned" model was compared with the original one by using Steiger's test and Bonferroni correction.

\subsection{Two connection patterns divided from the connectome model}

To better understand the relationships between PD motor impairment and various within- and betweenfunctional connections, we divided the connectome model into two connection patterns. One pattern that contained all consensus negative connections was called the "negative motor-impairment-related network", while another pattern that contained all consensus positive connections was called the " positive motor-impairment-related network". Characteristics of within- and between-network connections were analyzed by summing the consensus connections using the above-mentioned seven canonical functional networks in the positive and negative motor-impairment-related networks. To control the effect of network size variations, the proportion of the connections within and between networks was also calculated by the actual number of connections / total number of possible connections [17].

\section{Statistical analyses}

Clinical characteristics of drug-naïve and drug-managed patients were analyzed by using SPSS software (Version 25). Kolmogorov-Smirnov test was applied to identify the normal distribution of continuous variables. The difference of normally distributed variables between two groups was analyzed with the two-sample t-test, otherwise, the Mann-Whitney test was conducted. And differences between qualitative variables were compared with the Chi-square test. Statistical analyses of model construction, validation, and evaluation were performed by using MATLAB (R2020b, MathWorks) and had been detailed in corresponding parts mentioned above. A two-sided $p$-value $<0.05$ was considered significant unless mentioned.

\section{Results}

\section{Characteristic of enrolled patients}

A total of 162 PD patients were enrolled in this study, including 47 drug-naïve and 115 drug-managed patients. Characteristics of patients in these two groups were shown in Table1. Except the treatment status (LEDD and duration of treatment), drug-managed patients had a significantly longer disease duration ( $p=0.005)$, higher $\mathrm{H}-\mathrm{Y}$ stage $(\mathrm{p}<0.001)$, and higher UPDRS III score $(\mathrm{p}=0.002)$ compared with drugnaïve patients. No significant difference in age, sex, MMSE score, and education was observed between two groups. Spearman correlation analysis demonstrated UPDRS III scores of drug-naïve patients were significantly related with age $(r=0.404, p=0.004)$ and disease duration $(r=0.527, p<0.001)$, which had been controlled by partial analysis in the candidate connections selection process. 


\section{Constructing and evaluating model in drug-naïve patients}

\subsection{Optimal threshold for connections selection}

The optimal threshold for maximizing the $r_{\text {true }}$ of $M, M 1, M 2$, and $M 3$ was identified after repeating the model construction process across connection selection thresholds of $p$-values ranging from 0.05 to 0.001. Besides, the MSE value under each threshold within these four models was also calculated for further evaluation of the predictive accuracy. The optimal p-value thresholds of $M, M 1, M 2$, and $M 3$ were $0.009,0.008,0.001$ and 0.001 , respectively. In general, there appeared to be a consistent inverse relationship between $r_{\text {true }}$ and the MSE, which meant that the $p$-value associated with the highest $r_{\text {true }}$ value was highly similar to those associated with a small MSE value. The $r_{\text {true }}$ and MSE values across the tested range of $p$-value were shown in supplementary materials (Figure S2).

\subsection{Predictability evaluation and model comparison}

There were 115 consensus connections selected to construct model M, including 58 consensus negative connections and 57 consensus positive connections (Figure 2). Model M obtained the highest sum of selected connections, representing $0.32 \%$ of the sum of whole-brain 35778 connections. Significant correlation between observed and predicted UPDRS III scores demonstrated that the constructed model could predict individual motor impairment severity among drug-naïve PD patients $\left(r_{\text {true }}=0.845, p<0.001\right.$, $p_{\text {permu}}=0.002, M S E=137.57$, figure 3 , drug-naïve group).

Compared with the other three models, model $\mathrm{M}$ had a significantly higher $\mathrm{r}_{\text {true }}$ value after Steiger's $\mathrm{Z}$ test (detailed in table 2 and supplemental material, figure S3). Besides, model M contained all consensus connections selected by M1 and M2. These results suggested that model $\mathrm{M}$ offered more valuable information related to PD motor impairment, thus making it have a better predictive efficacy in the training set. Therefore, model $\mathrm{M}$ was employed in the subsequent analysis.

\subsection{Head-motion control}

Head-motion of each patient, which was evaluated by calculating mean FD, had no significant relationship with observed scores $(r=0.031, p=0.837)$ and the predicted scores generated from model $M$ $(r=0.045, p=0.762)$. Furthermore, combining the mean FD as an additional nuisance variable when selecting candidate connections, the constructed model remained predictable among drug-naïve patients $\left(r_{\text {true }}=0.835, p<0.001, p_{\text {permu }}=0.001, M S E=135.73\right)$. The predictability of this model was not significantly different from the original one (Steiger's $Z$ value $=1.936, p=0.053$ ). Besides, the common connections were highly overlapped with the network of model $\mathrm{M}$ after controlling for head-motion (percentage of overlapping: higher-UPDRS III score network 94.7\%, lower-UPDRS III score 91.4\%). These results suggested that head motion did not have significant confounding effects on our major results.

\section{Validating constructed model in drug-managed patients}


Model M significantly predicted UPDRS III score in the independent drug-managed group $(r=0.209$, $p=0.025, M S E=182.96$ ) (Figure 3). This result remained stable after adding head-motion as a nuisance variable in the candidate connection selection process $(r=0.219, p=0.019, \mathrm{MSE}=182.17)$ and did not significantly different from the original one (Steiger's $Z$ value $=0.626, p=0.535$ ). These results demonstrated that head-motion did not significantly affect the predictability of model $M$ in drug-managed patients. Furthermore, after controlling characteristics significantly differing from the drug-naïve group (including disease duration, $\mathrm{H}-\mathrm{Y}$ stage, LEDD, and treatment duration) within partial correlation analysis, predicted scores generated from model $\mathrm{M}$ remained significantly associated with the observed scores $(r=0.241, p=0.012)$.

\section{Analysis of Functional network anatomy}

\subsection{Contribution of each functional network to motor impairment prediction}

By summarizing consensus positive and negative connections together, we found that motor network contributed mostly, following with frontoparietal and limbic networks. After controlling the network sizes, results consistently showed that the motor, the limbic, and the frontoparietal networks were the top three ones contributing to the connectome model (detailed in supplementary materials, figure S4). Next, we tested the importance of each single functional network for motor impairment prediction by constructing lesioned models and the results were detailed in table 3. Comparing with the whole-brain connectome model, predictability of the lesioned model was significantly worse in the drug-naïve group after excluding frontoparietal network( Steiger's Z value=2.149,p=0.032) and visual-related network(Steiger's Z value=2.246, $p=0.025$ ), as well as in drug-managed group after excluding basial-ganglia network (Steiger's Z value=2.232, $\mathrm{p}=0.026$ ). While after excluding visual-related networks, the predictability tended to be improved among drug-managed group (Steiger's Z value=0.961, p=0.049). However, none of them survived Bonferroni correction. The results of the study demonstrate that the constructed model did not rely on strength in a single functional network, but rather incorporates motor-impairment-related information from various neural networks throughout the brain.

\subsection{Exploring connection patterns of the constructed model}

As we know that PD motor impairment is caused by the functional alternation of the whole-brain networks, we then explore connection patterns within and between the seven functional networks in the positive and negative motor-impairment-related networks by taking the network sizes into consideration and obtaining the proportion of each network (Figure 4).

Results showed that, in the negative motor-impairment-related network, connections within motor network, visual-related network, and default mode network were the top three networks that contributed to this pattern. It reflected that negative motor-impairment-related network had a more segregated pattern, which contained more within-network connections (within- vs. between- network connections: 26.35 vs. $20.57 \%$, detailed in supplemental materials Table S1). Whereas in the positive motor-impairment-related networks, connections between motor-frontoparietal, motor-basial ganglia, and motor-limbic networks 
contributed mostly.It indicated that positive motor-impairment-related network had a more integrated pattern, involving more between-network connections (within- vs. between- network connections: 5.02 vs. $28.62 \%$, detailed in supplemental materials, Table S1).

These results demonstrated that more within-network connections among motor, visual, and default mode networks indicated lower UPDRS III scores and mild PD motor impairment, while more betweennetwork connections among motor-frontoparietal, motor-basial ganglia, and motor-limbic networks were associated with higher UPDRS III scores and severe PD motor impairment.

\section{Discussion}

By applying the data-driven CPM method, a resting-state-functional connectome-based model was constructed to predict the severity of motor impairment in drug-naive patients, and demonstrate the characterizations of PD motor impairment as associated with coordinated functional activity over distinct networks. Moreover, this functional connectome-based model constructed in drug-naïve patients could predict motor impairment in drug-managed patients.

According to the correlation coefficient between their strength and UPDRS III scores, we assigned all selected connections into two patterns: negative and positive motor-impairment-related networks. The negative motor-impairment-related network, which enrolled all negative connections, contained more within-network connections, reflecting higher segregation of distinct networks; while the positive motorimpairment-related network, which enrolled all consensus positive connections, contained more betweennetwork connections suggesting highly integrated communication across different district networks [32, 33]. Consistent with our findings, Kim et al. demonstrated PD patients were less likely to be in segregated states when compared with healthy aging, and higher UPDRS III scores were related to a shorter dwell time of segregated state and a longer dwell time of integration state [34,35]. Therefore, our study suggested that, the decreased functional segregation and increased functional integration may play a critical role in modulating motor impairments in PD.

Then, we investigated the predictive contribution of each functional network in negative motorimpairment-related network, which was mainly composed of connections within the motor network, visual network, and default mode network. Thus, connection disruption of these three networks may deteriorate motor impairment in PD. Furthermore, among these connections, motor network occupied the most important place. And comparably, a number of previous studies also suggested that intrinsic dysfunction of the motor network had a close relationship with motor deterioration in PD [36-38], even in PD patients at different disease stages [14]. In addition to motor network, visual-related network and default mode network were shown to contribute significantly to the negative motor-impairment-related network. Visual dysfunction is one of the major symptoms in PD, including loss of visual acuity and color vision as well as higher-order visual deficits [39]. The interaction between visual and motor networks is important for move control and move learning[40] and has been confirmed to be impaired in PD patients [41]. Besides, a significant negative correlation between connections' strength within visual network and motor 
symptom severity had been demonstrated among PD patients with freezing of gait [10]. Default mode network is the most studied network with the highest network connections and complicated neural modulations [42]. Specifically, a couple of studies demonstrated that default mode network participants in motor coordination and cognitive function, both of which are closely related with motor modulation [43, 44]. And pathologically, the $\alpha$-synuclein deposition along with the disruption of dopaminergic pathway could affect the modulation between the DMN activity and other networks, leading to motor impairment[45]. Taken together, this negative motor-impairment-related network, composed mostly by within-network connections of motor, visual and default mode networks, provides insightful evidence that motor impairment in PD was caused by dysfunctional of these networks, which could not simply be blamed on the motor network.

On the other side, the positive motor-impairment-related network was mainly composed of connections between motor-frontoparietal, motor-basial ganglia, and motor-limbic networks. Therefore, based on the widespread disruption of motor network in PD as mentioned above, the increased connections of motor network with other networks, e.g., frontoparietal, basal ganglia, and limbic networks, may indicate their enhanced activation to protect motor network from pathophysiological dysfunction, leading to an amplified motor modulation. Other than the enhanced connection between motor and basial-ganglia networks that have been demonstrated associating with motor impairment severity [14,46], a potential compensation mechanism of the brain to overcome motor impairment by increasing the communication between motor-frontoparietal networks and motor-limbic networks has also been identified in this study. Increased functional activity within frontoparietal and limbic networks has been reported to be related to PD motor symptoms such as hypokinesia/akinesia [47], freezing of gait [48, 49], "masked face" syndrome [50]. The frontoparietal network is considered to be one of the top-down control networks involving in initiate and adjust control[51]. Meanwhile, the limbic-motor connections were also reported to be involved in the emotional adjustment of complex functions like spatial perception and movement computation[50]. The enhanced functional communication of motor-frontoparietal networks and motorlimbic networks detected in positive motor-impairment-related network reflected that PD patients may rely on more attentional and emotional resources to operate their motor dysfunction due to a loss of automaticity.

Since PD is clinically heterogeneous and drug uptake would influence the brain organization, it deserves to validate the constructed CPM model in drug-managed patients. As a result, the significant prediction was observed after translating the CPM model constructed on drug-naïve patients to drug-managed patients. Although all drug-managed patients were required to wash out drugs for $12 \mathrm{~h}$, the long-duration response of levodopa can persist for several days after stopping taking drugs, which has been demonstrated that could affect cortical function involving motor control $[52,53]$. The successful validation in drug-managed patients demonstrated that these selected functional connectome representing the intrinsic organization of PD preserved after chronic levodopa treatment. Although withinsubject comparisons between pre-and post-treatment status would be a stronger test of this hypothesis, the current results provide compelling evidence that these generalizable brain-behavior associations were independent of the effect of dopamine. In summary, our findings suggested that these disease-intrinsic 
connectome characteristics identified in drug-naïve patients at the individual level had potential to be a stable biomarker for PD motor impairment severity.

This study has several limitations that should be acknowledged. First, most patients enrolled in this study were at a relative early stage, therefore the model still needs to be validated among advanced PD patients in the future study. Second, the current results were obtained from a cross-sectional study, a longitudinal study to validate the connectome model and track their progression would be necessary for the future. Third, as the previous study suggested, multimodal brain data could be applied in further study, which might improve the predictability[54].

\section{Conclusions}

In conclusion, this study identified a whole-brain connectome-based model that could predict the severity of motor impairment among drug-naïve patients, which can be further applied in an independent drugmanaged group. The connection patterns generated from our model suggested that functional segregation of motor, default mode, and visual-related networks play an important role in PD motor impairment, and higher connections coupling motor-frontoparietal, motor-basial ganglia, and motorlimbic networks might demonstrate a compensatory mechanism to overcome motor dysfunction. This generalizable brain-behavior association can be detected in relation to pre-and post-treatment, indicating a relatively stable way to estimate motor impairment severity among PD.

\section{Abbreviations}

PD: Parkinson's disease; CPM:Connectome-based predictive model; UPDRS III:Unified Parkinson's Disease Rating Scale part III; MSE: Mean squared error; rs-fMRI: Resting-state functional magnetic resonance imaging; MMSE: Mini-Mental State Examination; $\mathrm{H}-\mathrm{Y}$ stage: Hoehn and Yahr stage; ;LEDD: Levodopa equivalent daily dose; FD: Frame-wise displacement (FD); LOOCV: Leave-one-out cross-validation

\section{Declarations}

\section{Ethics approval and consent to participate}

All PD patients signed informed consent forms and all procedures performed in studies involving human participants were in accordance with the ethical standards of the institutional research committee and with the 1964 Helsinki declaration and its later amendments or comparable ethical standards.

\section{Consent for publication}

Not applicable.

\section{Availability of data and materials}


The materials used and/or analyzed during the current study are available from the corresponding author on reasonable request.

\section{Competing interests}

The authors declare that they have no competing interests

\section{Funding}

This work was supported by the 13th Five-year Plan for National Key Research and Development Program of China (Grant No. 2016YFC1306600), the National Natural Science Foundation of China (Grant Nos. 82001767, 81971577, 81771820), the Natural Science Foundation of Zhejiang Province (Grant No. LQ21H180008, LQ20H180012), the China Postdoctoral Science Foundation (Grant No. 2021T140599 and 2019M662082), the National Natural Science Foundation of China's Major Regional International Cooperation Project (Grant No. 81520108010), and the Key Research and Development Program of Zhejiang Province (Grant No. 2020C03020).

\section{Authors' contributions}

All of the coauthors listed meet the criteria for authorship. HTW was involved with study concept and design, acquisition of data, analysis and interpretation of data, drafting/revising the manuscript. XQB, $X C L$, JWC, JQW, TG, and LYG were involved with acquisition of data, analysis and interpretation of data. $\mathrm{CZ}, \mathrm{GT}$, and JJWwere involved with data analysis and manuscript revision. XJG, XJX, and PYH was responsible for data interpretation, manuscript revision, obtaining funding and study supervision. BRZ were involved with PD patients' recruitment. MMZ was responsible for study concept, revising the manuscript, obtaining funding and study supervision. All authors read and approved the final manuscript.

\section{Acknowledgements}

Not applicable.

\section{References}

1. Hayes MT: Parkinson's Disease and Parkinsonism. The American journal of medicine 2019, 132(7):802-807.

2. Kalia LV, Lang AE: Parkinson's disease. Lancet (London, England) 2015, 386(9996):896-912.

3. Kalia LV, Brotchie JM, Fox SH: Novel nondopaminergic targets for motor features of Parkinson's disease: review of recent trials. Movement disorders : official journal of the Movement Disorder Society 2013, 28(2):131-144.

4. Bartels AL, Leenders KL: Parkinson's disease: the syndrome, the pathogenesis and pathophysiology. Cortex; a journal devoted to the study of the nervous system and behavior 2009, 45(8):915-921. 
5. Guan X, Zhang Y, Wei H, Guo T, Zeng Q, Zhou C, Wang J, Gao T, Xuan M, Gu Q et al: Iron-related nigral degeneration influences functional topology mediated by striatal dysfunction in Parkinson's disease. Neurobiology of aging 2019, 75:83-97.

6. Hacker CD, Perlmutter JS, Criswell SR, Ances BM, Snyder AZ: Resting state functional connectivity of the striatum in Parkinson's disease. Brain : a journal of neurology 2012, 135(Pt 12):3699-3711.

7. Nachev P, Kennard C, Husain M: Functional role of the supplementary and pre-supplementary motor areas. Nature reviews Neuroscience 2008, 9(11):856-869.

8. Kann SJ, Chang C, Manza P, Leung HC: Akinetic rigid symptoms are associated with decline in a cortical motor network in Parkinson's disease. NPJ Parkinson's disease 2020, 6:19.

9. Vervoort G, Alaerts K, Bengevoord A, Nackaerts E, Heremans E, Vandenberghe W, Nieuwboer A: Functional connectivity alterations in the motor and fronto-parietal network relate to behavioral heterogeneity in Parkinson's disease. Parkinsonism \& related disorders 2016, 24:48-55.

10. Tessitore A, Amboni M, Esposito F, Russo A, Picillo M, Marcuccio L, Pellecchia MT, Vitale C, Cirillo M, Tedeschi $\mathrm{G}$ et al: Resting-state brain connectivity in patients with Parkinson's disease and freezing of gait. Parkinsonism \& related disorders 2012, 18(6):781-787.

11. Gilat M, Ehgoetz Martens KA, Miranda-Domínguez O, Arpan I, Shine JM, Mancini M, Fair DA, Lewis SJG, Horak FB: Dysfunctional Limbic Circuitry Underlying Freezing of Gait in Parkinson's Disease. Neuroscience 2018, 374:119-132.

12. Tahmasian M, Bettray LM, van Eimeren T, Drzezga A, Timmermann L, Eickhoff CR, Eickhoff SB, Eggers C: A systematic review on the applications of resting-state fMRI in Parkinson's disease: Does dopamine replacement therapy play a role?Cortex; a journal devoted to the study of the nervous system and behavior 2015, 73:80-105.

13. Krismer F, Seppi K: The Parkinson disease connectome - insights from new imaging studies. Nature reviews Neurology 2021.

14. Tessitore A, Cirillo M, De Micco R: Functional Connectivity Signatures of Parkinson's Disease. Journal of Parkinson's disease 2019, 9(4):637-652.

15. Ren Z, Daker RJ, Shi L, Sun J, Beaty RE, Wu X, Chen Q, Yang W, Lyons IM, Green AE et al: Connectome-Based Predictive Modeling of Creativity Anxiety. Neurolmage 2021, 225:117469.

16. Yu J, Rawtaer I, Fam J, Feng L, Kua EH, Mahendran R: The individualized prediction of cognitive test scores in mild cognitive impairment using structural and functional connectivity features. Neurolmage 2020, 223:117310.

17. Gao M, Wong CHY, Huang H, Shao R, Huang R, Chan CCH, Lee TMC: Connectome-based models can predict processing speed in older adults. Neurolmage 2020, 223:117290.

18. Shang R, He L, Ma X, Ma Y, Li X: Connectome-Based Model Predicts Deep Brain Stimulation Outcome in Parkinson's Disease. Frontiers in computational neuroscience 2020, 14:571527.

19. Hughes AJ, Daniel SE, Kilford L, Lees AJ: Accuracy of clinical diagnosis of idiopathic Parkinson's disease: a clinico-pathological study of 100 cases. Journal of neurology, neurosurgery, and psychiatry 1992, 55(3):181-184. 
20. Zhang MY, Katzman R, Salmon D, Jin H, Cai GJ, Wang ZY, Qu GY, Grant I, Yu E, Levy P et al: The prevalence of dementia and Alzheimer's disease in Shanghai, China: impact of age, gender, and education. Annals of neurology 1990, 27(4):428-437.

21. Katzman R, Zhang MY, Ouang Ya Q, Wang ZY, Liu WT, Yu E, Wong SC, Salmon DP, Grant I: A Chinese version of the Mini-Mental State Examination; impact of illiteracy in a Shanghai dementia survey. Journal of clinical epidemiology 1988, 41(10):971-978.

22. Tomlinson CL, Stowe R, Patel S, Rick C, Gray R, Clarke CE: Systematic review of levodopa dose equivalency reporting in Parkinson's disease. Movement disorders : official journal of the Movement Disorder Society 2010, 25(15):2649-2653.

23. Yan CG, Wang XD, Zuo XN, Zang YF: DPABI: Data Processing \& Analysis for (Resting-State) Brain Imaging. Neuroinformatics 2016, 14(3):339-351.

24. Jenkinson M, Bannister P, Brady M, Smith S: Improved optimization for the robust and accurate linear registration and motion correction of brain images. Neurolmage 2002, 17(2):825-841.

25. Yan CG, Cheung B, Kelly C, Colcombe S, Craddock RC, Di Martino A, Li Q, Zuo XN, Castellanos FX, Milham MP: A comprehensive assessment of regional variation in the impact of head micromovements on functional connectomics. Neurolmage 2013, 76:183-201.

26. Shen X, Tokoglu F, Papademetris X, Constable RT: Groupwise whole-brain parcellation from restingstate fMRI data for network node identification. Neurolmage 2013, 82:403-415.

27. Wang J, Wang X, Xia M, Liao X, Evans A, He Y: GRETNA: a graph theoretical network analysis toolbox for imaging connectomics. Frontiers in human neuroscience 2015, 9:386.

28. Scheinost D, Noble S, Horien C, Greene AS, Lake EM, Salehi M, Gao S, Shen X, O'Connor D, Barron DS et al: Ten simple rules for predictive modeling of individual differences in neuroimaging. Neurolmage 2019, 193:35-45.

29. Rosenberg MD, Finn ES, Scheinost D, Papademetris X, Shen X, Constable RT, Chun MM: A neuromarker of sustained attention from whole-brain functional connectivity. Nature neuroscience 2016, 19(1):165-171.

30. Shen X, Finn ES, Scheinost D, Rosenberg MD, Chun MM, Papademetris X, Constable RT: Using connectome-based predictive modeling to predict individual behavior from brain connectivity. Nature protocols 2017, 12(3):506-518.

31. Lake EMR, Finn ES, Noble SM, Vanderwal T, Shen X, Rosenberg MD, Spann MN, Chun MM, Scheinost D, Constable RT: The Functional Brain Organization of an Individual Allows Prediction of Measures of Social Abilities Transdiagnostically in Autism and Attention-Deficit/Hyperactivity Disorder. Biological psychiatry 2019, 86(4):315-326.

32. King BR, van Ruitenbeek P, Leunissen I, Cuypers K, Heise KF, Santos Monteiro T, Hermans L, Levin O, Albouy G, Mantini D et al: Age-Related Declines in Motor Performance are Associated With Decreased Segregation of Large-Scale Resting State Brain Networks. Cerebral cortex (New York, NY : 1991) 2018, 28(12):4390-4402. 
33. Damoiseaux JS: Effects of aging on functional and structural brain connectivity. Neurolmage 2017, 160:32-40.

34. Kim J, Criaud M, Cho SS, Díez-Cirarda M, Mihaescu A, Coakeley S, Ghadery C, Valli M, Jacobs MF, Houle $S$ et al: Abnormal intrinsic brain functional network dynamics in Parkinson's disease. Brain : a journal of neurology 2017, 140(11):2955-2967.

35. Nieuwhof F, Helmich RC: Entangled cerebral networks in Parkinson's disease. Brain : a journal of neurology 2017, 140(11):2767-2769.

36. Lewis GN, Byblow WD: Altered sensorimotor integration in Parkinson's disease. Brain : a journal of neurology 2002, 125(Pt 9):2089-2099.

37. Tessitore A, Giordano A, De Micco R, Russo A, Tedeschi G: Sensorimotor connectivity in Parkinson's disease: the role of functional neuroimaging. Frontiers in neurology 2014, 5:180.

38. Wu T, Wang L, Chen Y, Zhao C, Li K, Chan P: Changes of functional connectivity of the motor network in the resting state in Parkinson's disease. Neuroscience letters 2009, 460(1):6-10.

39. Weil RS, Schrag AE, Warren JD, Crutch SJ, Lees AJ, Morris HR: Visual dysfunction in Parkinson's disease. Brain : a journal of neurology 2016, 139(11):2827-2843.

40. Glickstein M: How are visual areas of the brain connected to motor areas for the sensory guidance of movement? Trends in neurosciences 2000, 23(12):613-617.

41. Inzelberg R, Schechtman E, Hocherman S: Visuo-motor coordination deficits and motor impairments in Parkinson's disease. PloS one 2008, 3(11):e3663.

42. Mohan A, Roberto AJ, Mohan A, Lorenzo A, Jones K, Carney MJ, Liogier-Weyback L, Hwang S, Lapidus KA: The Significance of the Default Mode Network (DMN) in Neurological and Neuropsychiatric Disorders: A Review. The Yale journal of biology and medicine 2016, 89(1):49-57.

43. Greicius MD, Krasnow B, Reiss AL, Menon V: Functional connectivity in the resting brain: a network analysis of the default mode hypothesis. Proceedings of the National Academy of Sciences of the United States of America 2003, 100(1):253-258.

44. Fox MD, Raichle ME: Spontaneous fluctuations in brain activity observed with functional magnetic resonance imaging. Nature reviews Neuroscience 2007, 8(9):700-711.

45. Christopher L, Duff-Canning S, Koshimori Y, Segura B, Boileau I, Chen R, Lang AE, Houle S, Rusjan P, Strafella AP: Salience network and parahippocampal dopamine dysfunction in memory-impaired Parkinson disease. Annals of neurology 2015, 77(2):269-280.

46. Kwak Y, Peltier S, Bohnen NI, Müller ML, Dayalu P, Seidler RD: Altered resting state cortico-striatal connectivity in mild to moderate stage Parkinson's disease. Frontiers in systems neuroscience 2010, 4:143.

47. Martin JA, Zimmermann N, Scheef L, Jankowski J, Paus S, Schild HH, Klockgether T, Boecker H: Disentangling motor planning and motor execution in unmedicated de novo Parkinson's disease patients: An fMRI study. Neurolmage Clinical2019, 22:101784. 
48. Bartels AL, Leenders KL: Brain imaging in patients with freezing of gait. Movement disorders : official journal of the Movement Disorder Society 2008, 23 Suppl 2:S461-467.

49. Shine JM, Matar E, Ward PB, Bolitho SJ, Gilat M, Pearson M, Naismith SL, Lewis SJ: Exploring the cortical and subcortical functional magnetic resonance imaging changes associated with freezing in Parkinson's disease. Brain : a journal of neurology 2013, 136(Pt 4):1204-1215.

50. Rizzo G, Milardi D, Bertino S, Basile GA, Di Mauro D, Calamuneri A, Chillemi G, Silvestri G, Anastasi G, Bramanti A et al: The Limbic and Sensorimotor Pathways of the Human Amygdala: A Structural Connectivity Study. Neuroscience 2018, 385:166-180.

51. Dosenbach NU, Fair DA, Cohen AL, Schlaggar BL, Petersen SE: A dual-networks architecture of topdown control. Trends in cognitive sciences 2008, 12(3):99-105.

52. Cilia R, Cereda E, Akpalu A, Sarfo FS, Cham M, Laryea R, Obese V, Oppon K, Del Sorbo F, Bonvegna S et al: Natural history of motor symptoms in Parkinson's disease and the long-duration response to levodopa. Brain : a journal of neurology 2020, 143(8):2490-2501.

53. Donzuso G, Sciacca G, Rascunà C, Cicero CE, Mostile G, Nicoletti A, Zappia M: Structural MRI substrate of long-duration response to levodopa in Parkinson's disease: an exploratory study. Journal of neurology 2021.

54. Helmich RC, Vaillancourt DE, Brooks DJ: The Future of Brain Imaging in Parkinson's Disease. Journal of Parkinson's disease 2018, 8(s1):S47-s51.

\section{Tables}

Table 1 Patients' Characteristic 


\begin{tabular}{|c|c|c|c|}
\hline Patient's characteristic & $\begin{array}{l}\text { Drug-naïve group } \\
(\mathrm{N}=47)\end{array}$ & $\begin{array}{l}\text { Drug managed group } \\
(\mathrm{N}=115)\end{array}$ & $p$-value \\
\hline $\begin{array}{l}\text { Sex } \\
\text { (Male/Female) }\end{array}$ & $22: 25$ & $65: 50$ & 0.061 \\
\hline $\begin{array}{l}\text { Age } \\
\text { (years, mean ) }\end{array}$ & 57.7210 .085 & 60.449 .581 & 0.109 \\
\hline $\begin{array}{l}\text { Disease duration } \\
\text { (years, median(range)) }\end{array}$ & $1.82(0.08-10.25)$ & $3.47(0.08-26.37)$ & $0.005^{*}$ \\
\hline $\begin{array}{l}\text { UPDRS III score } \\
\text { (median(range)) }\end{array}$ & $18(4-68)$ & 19(3-66) & $0.002^{*}$ \\
\hline $\begin{array}{l}\mathrm{H}-\mathrm{Y} \text { stage } \\
\text { (median(range)) }\end{array}$ & $2(1-3)$ & $2.5(1-5)$ & $<0.001^{\star}$ \\
\hline $\begin{array}{l}\text { LEED } \\
\text { (median(range)) }\end{array}$ & - & $425(25-1300)$ & NA \\
\hline $\begin{array}{l}\text { Treatment Duration } \\
\text { (years, median(range)) }\end{array}$ & - & $2.04(0.04-26.37)$ & NA \\
\hline $\begin{array}{l}\text { MMSE } \\
\text { (median(range)) }\end{array}$ & $28(19-30)$ & $28(17-30)$ & 0.497 \\
\hline $\begin{array}{l}\text { Education } \\
\text { (median(range)) }\end{array}$ & $9(0-18)$ & $9(0-18)$ & 0.459 \\
\hline $\begin{array}{l}\text { Notes: } p<0.05 \text { was cons } \\
\text { Abbreviations: UPDRS II } \\
\text { Yahr stage; LEDD: levod }\end{array}$ & $\begin{array}{l}\text { statistically signifi } \\
\text { ified Parkinson's D } \\
\text { valent daily dose; } ~\end{array}$ & $\begin{array}{l}\text { nnotated with *). } \\
\text { Rating Scale part III; H- } \\
\text { available; }\end{array}$ & ehn and \\
\hline
\end{tabular}

Table2 Predictability of connectome-based models and comparison 


\begin{tabular}{|c|c|c|c|c|}
\hline Different Models & $r_{\text {true }}\left(p, p_{\text {permu }}\right)$ & MSE & Steiger's Z value & p-value \\
\hline Model M & $0.845(<0.001,0.002)$ & 137.57 & - & - \\
\hline Model M1 & $0.712(<0.001,0.014)$ & 182.83 & 2.63 & $0.008^{\star}$ \\
\hline Model M2 & $0.711(<0.001,0.012)$ & 159.95 & 2.39 & $0.017^{*}$ \\
\hline Model M3 & $0.528(<0.001,0.002)$ & 248.69 & 4.44 & $<0.001^{*}$ \\
\hline \multicolumn{5}{|c|}{$\begin{array}{l}\text { Notes: } r_{\text {true }} \text { : the true predictive correlation coefficient between observed and predict scores; } p_{\text {permu: }} p^{-} \\
\text {value obtained from permutation test (1000 times); } p<0.05 \text { was considered as statistically significant } \\
\text { (annotated with *). }\end{array}$} \\
\hline \multicolumn{5}{|c|}{ Abbreviations: MSE: mean squared error. } \\
\hline
\end{tabular}

Table 3 Predictability of lesioned models constructed from drug-naïve patients and validated on drugmanaged patients.

\begin{tabular}{|c|c|c|c|c|c|}
\hline & & \multicolumn{2}{|c|}{ Drug-naïve group } & \multicolumn{2}{|c|}{ Drug-managed group } \\
\hline & & $r, p$ & $\mathrm{Z}, \mathrm{p}$ & $r, p$ & $Z, p$ \\
\hline & Whole brain & $0.845,<0.001$ & - & $0.208,0.025$ & - \\
\hline \multirow[t]{7}{*}{ Lesioned models } & -FP & $0.811,<0.001$ & $2.149,0.032$ & $0.202,0.031$ & $0.205,0.837$ \\
\hline & -DM & $0.845,<0.001$ & $0,1.0$ & $0.202,0.030$ & $0.535,0.592$ \\
\hline & - Mot & $0.809,<0.001$ & $1.426,0.154$ & $0.147,0.117$ & $1.110,0.267$ \\
\hline & -Vis & $0.819,<0.001$ & $2.246,0.025$ & $0.209,0.024$ & $0.961,0.049$ \\
\hline & -Lim & $0.836,<0.001$ & $0.587,0.557$ & $0.195,0.036$ & $0.639,0.523$ \\
\hline & $-B G$ & $0.845,<0.001$ & $0,1.0$ & $0.192,0.039$ & $2.232,0.026$ \\
\hline & -Cer & $0.843,<0.001$ & $0.695,0.392$ & $0.203,0.029$ & $0.838,0.402$ \\
\hline \multicolumn{6}{|c|}{$\begin{array}{l}\text { Note冈Predictability of models constructed from drug-naïve patients were generated with consensus } \\
\text { connections retained significant by using the optimal threshold of model } \mathrm{M}(\mathrm{p}=0.009) \text {. Predictability } \\
\text { was assessed with Spearman correlation coefficient (r) between observed and predicted scores. } \\
\text { Predictability from whole-brain matrix is included in the first row for comparison. The predictability of } \\
\text { lesioned model was compared with model M (using whole-brain matrix) by applying Steiger's Z test } \\
\text { and Bonferroni correction. None of these models survive Bonferroni correction. } \\
\text { Abbreviations: FP: frontoparietal network; DMN: Default mode network; Mot: motor network; Vis: } \\
\text { visual-related network; Lim: limbic network; BG: basial-ganglia network; Cer: cerebellum network; }\end{array}$} \\
\hline
\end{tabular}




\section{Figures}

Functional network construction

Model construction and evaluation in drug-naïve patients

\section{Predictability comparison with} models constructed with different methods

\section{Validation in drug-managed} patients
Connection selection by using LOOCV procedure

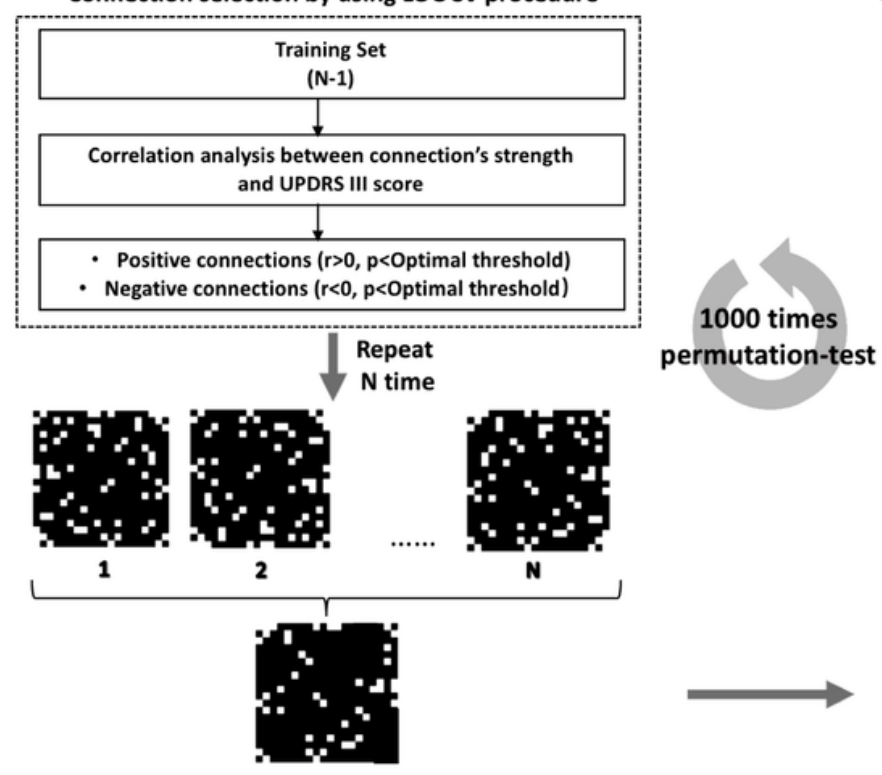

Predictive model constructed with consensus connections
Apply model to generate predicted score

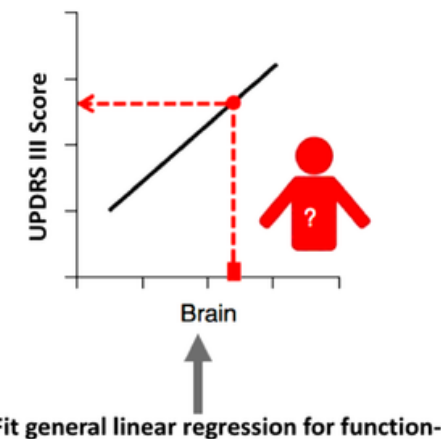
UPDRS III score relationship

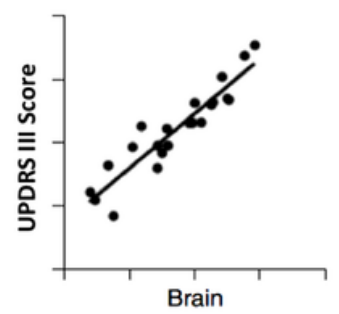

\section{Figure 1}

Flowchart for identifying a whole-brain connectome-based model for predicting motor impairment in PD. The model $(M)$ was first constructed and evaluated among drug-naïve PD patients. Its predictive performance was further validated among drug-managed PD patients for reliability checking Abbreviations: UPDRS III: The Unified Parkinson's Disease Rating Scale part III, PD: Parkinson's disease 

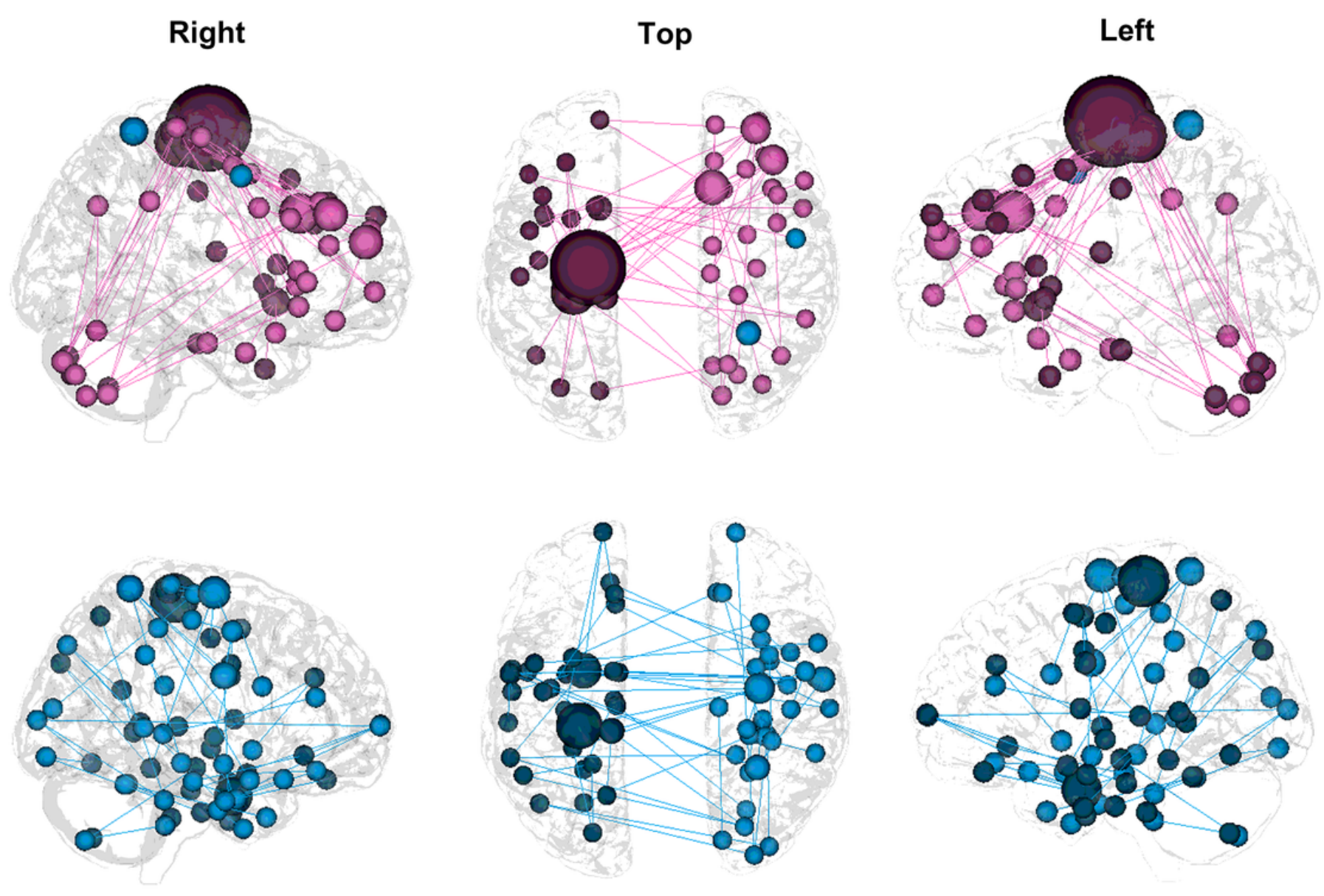

Figure 2

The constructed model (M) contained 58 consensus negative connections (blue) and 57 consensus positive connections (pink). Connectivity figures were created using the tool in the web browser (http://bisweb.yale.edu/connviewer/). 
Drug-naive group

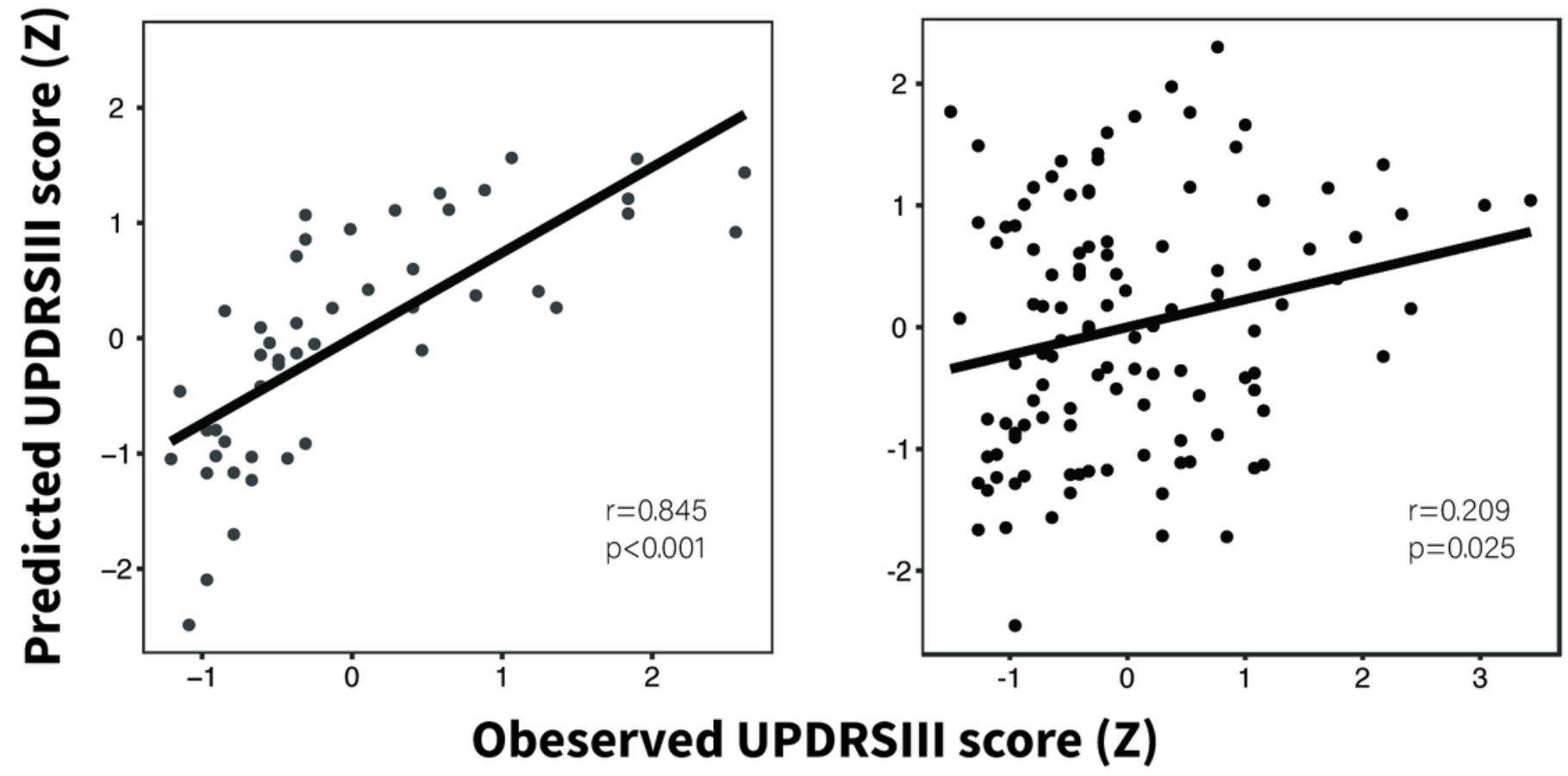

Figure 3

The constructed model $(\mathrm{M})$ could predict individual motor impairment severity of drug-naïve and drugmanaged patients. Both predicted and observed scores were standardized for visualization. Abbreviations: UPDRS III: The Unified Parkinson's Disease Rating Scale part III

Negative motor-impairment-related network

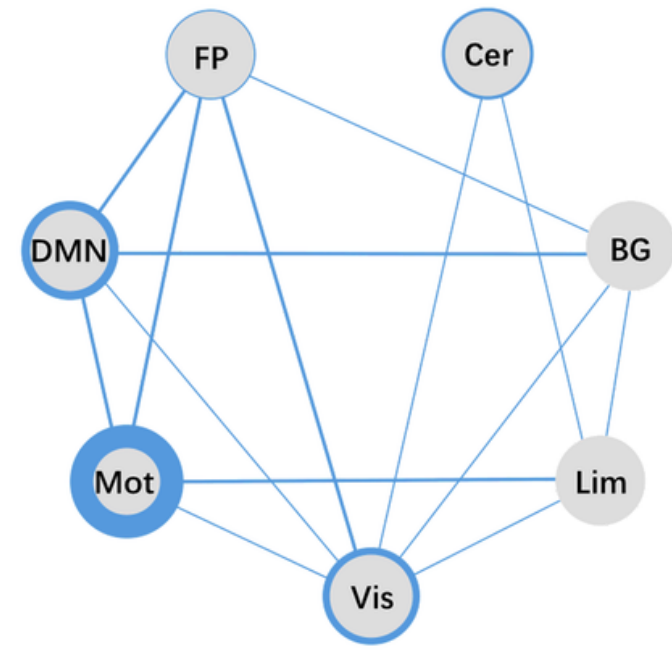

Positive motor-impairment-related network

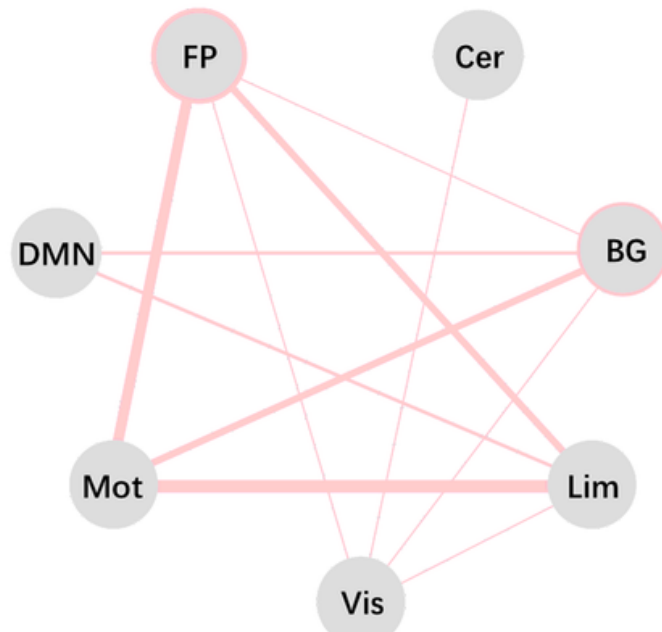

Figure 4

Negative (left, blue) and positive motor-impairment-related networks (right, pink). To control the possible effect of network size, the proportion of the within- and between-network connection is obtained by 
dividing the actual number of connections by the total number of all possible connections. Each solid circle represents a functional network, and thicker circles and lines represent a greater proportion of connectivity. Abbreviations: FP: frontoparietal network; DMN: Default mode network; Mot: motor network; Vis: visual-related network; Lim: limbic network; BG: basial-ganglia network; Cer: cerebellum network;

\section{Supplementary Files}

This is a list of supplementary files associated with this preprint. Click to download.

- SupplementalMaterials.docx 